\title{
ON THE PATHS WITH MONGE'S EQUATIONS OF THE SECOND DEGREE AS CONDITIONS OF INTERSECTION
}

\author{
HSIEN-CHUNG WANG ${ }^{1}$
}

1. Introduction. The problem on the conditions of intersection of a family of curves was suggested by $\mathrm{S}$. $\mathrm{Lie}^{2}$ and systematically studied by $\mathrm{F}$. Engel. ${ }^{3}$ Under the latter's initiative extensive researches have been made on the subject. ${ }^{4}$ It is the aim of the present paper to point out the relations between this type of problems and the geometry of paths, thus obtaining the geometrical interpretation of some im-• portant invariants of a system of paths. To be definite, we consider in an $(n+1)$-dimensional space a $2 n$-parameter family of paths. By using Pfaffian forms, we can define the tensor of the lowest order of the paths in a simple way. This tensor is essentially the one which plays a rôle in the so-called inverse problem of the calculus of variations. ${ }^{5}$ It turns out that its vanishing is a necessary and sufficient condition for the conditions of intersection of neighboring paths to be given by a system of Monge's equations of the second degree.

2. The tensor. Let $\left(x, y^{i}\right)(i=1, \cdots, n)$ be coordinates of an $(n+1)$-dimensional space. A $2 n$-parameter family of paths is defined by a system of ordinary differential equations of the form

(1) $y^{i \prime \prime}=F^{i}\left(x, y^{j}, y^{j^{\prime}}\right), \quad i, j=1, \cdots, n, \quad y^{i \prime}=\frac{d y^{i}}{d x}, y^{i \prime \prime}=\frac{d^{2} y^{i}}{d x^{2}}$,

which can also be written in a Pfaffian system

$$
d y^{i}-p^{i} d x=0, \quad d p^{i}-F^{i}\left(x, y^{j}, p^{i}\right) d x=0,
$$

where $p^{i}$ are auxiliary variables. To simplify our calculations we introduce the following Pfaffian forms

Received by the editors February 9, 1944.

1 The author wishes to express his thanks to Professor Shiing-shen Chern for suggesting this problem.

2 S. Lie and G. Scheffers, Geometrie der Berihhrungstransformationen, vol. I, Leipzig, 1896, p. 269.

${ }^{8} \mathrm{~F}$. Engel, Eine neue Methode zur Invariantentheorie der Differentialgleichungen, Berichte uber die Verhandlungen der Königlich Sachsischen Gesellschaft der Wissenschaften vol. 57 (1905) pp. 161-232.

4 P. Steinbach, Pfaffsche Gleichungen als Schnittbedingung von Kurvenscharen, Mitteilungen des Mathematischen Seminars der Universität Giessen, vol. 2, no. 18, 1930.

J. Douglas, Solution of the inverse problem of the calculus of variations, Proc. Nat. Acad. Sci. U.S.A. vol. 25 (1939) pp. 631-637. 


$$
\begin{aligned}
\omega^{i} & =d y^{i}-p^{i} d x, \\
\pi^{i} & =d p^{i}-F^{i} d x-\left(\partial F^{i} / \partial p^{b}+\delta_{k}^{i} H\right)\left(d y^{k}-p^{k} d x\right) / 2,
\end{aligned}
$$

the function $H=H\left(x, y^{j}, p^{j}\right)$ being any particular solution of the partial differential equation

$$
2 n \frac{d H}{d x}+n H^{2}-4 \frac{\partial F^{i}}{\partial y^{i}}-\frac{\partial F^{i}}{\partial p^{i}} \frac{\partial F^{i}}{\partial p^{i}}+2 \frac{d}{d x}\left(\frac{\partial F^{i}}{\partial p^{i}}\right)=0 .
$$

Here, as throughout this paper, Latin indices run from 1 to $n$, repeated indices denote summation, and the operator $d / d x$ denotes total differentiation with respect to $x$ along any of the paths, that is,

$$
d / d x=\partial / \partial x+p^{i \partial} / \partial y^{i}+F^{i} \partial / \partial p^{i} .
$$

By a simple calculation we find ${ }^{6}$

where

$$
\begin{aligned}
\left(\omega^{i}\right)^{\prime}= & {\left[d x \pi^{i}\right]+\frac{1}{2}\left(\frac{\partial F^{i}}{\partial p^{k}}+\delta_{k}^{i} H\right)\left[d x \omega^{k}\right], } \\
\left(\pi^{i}\right)^{\prime}= & Q_{j}^{i}\left[d x \omega^{i}\right]+\frac{1}{2}\left(\frac{\partial F^{i}}{\partial p^{i}}-\delta_{j}^{i} H\right)\left[d x \pi^{i}\right] \\
& +\frac{1}{2}\left(\frac{\partial^{2} F^{i}}{\partial p^{k} \partial p^{l}}+\delta_{k}^{i} \frac{\partial H}{\partial p^{l}}\right)\left[\omega^{k} \pi^{l}\right]+Q_{j k}^{i}\left[\omega^{i} \omega^{k}\right],
\end{aligned}
$$

$$
\begin{aligned}
Q_{i}^{i}= & \frac{\partial F^{i}}{\partial y^{j}}+\frac{1}{4} \frac{\partial F^{i}}{\partial p^{k}} \frac{\partial F^{k}}{\partial p^{j}}-\frac{1}{2} \frac{d}{d x}\left(\frac{\partial F^{i}}{\partial p^{i}}\right) \\
& -\frac{1}{n} \delta_{j}^{i}\left\{\frac{\partial F^{m}}{\partial y^{m}}+\frac{1}{4} \frac{\partial F^{m}}{\partial p^{l}} \frac{\partial F^{l}}{\partial p^{m}}-\frac{1}{2} \frac{d}{d x} \frac{\partial F^{m}}{\partial p^{m}}\right\},
\end{aligned}
$$

while $Q_{j \mathrm{k}}^{i}$ are functions formed from $F^{i}$ and $H$ whose expressions do not interest us. The $n^{2}$ quantities $Q_{j}^{q}$ constitute a tensor in the following sense. Let

$$
\bar{x}=\bar{x}\left(x, y^{j}\right), \quad \bar{y}^{i}=\bar{y}^{i}\left(x, y^{j}\right)
$$

be a point transformation which carries our paths to a system of paths of the space $\left(\bar{x}, \bar{y}^{i}\right)$. Suppose the new paths be defined by the Pfaffian system

$$
d \bar{y}^{i}-\bar{p}^{i} d \bar{x}=0, \quad d \bar{p}^{i}-\bar{F}^{i}\left(\bar{x}, \bar{y}^{i}, \bar{p}^{j}\right) d \bar{x}=0 .
$$

- E. Goursat, Leçons sur le problème de Pfaff, Paris, 1922, pp. 15-20. 
from which the forms $\bar{\omega}^{i}, \bar{\pi}^{i}$ and the functions $\bar{H}, \bar{Q}_{j}^{i}$ can be defined in a similar way. Under this assumption there exist functions $u, u_{i}, u_{j}^{j}, r_{j}^{4}, s_{j}^{s}$ such that

$$
\begin{aligned}
d \bar{x} & =u d x+u_{i} \omega^{i}, \\
\bar{\omega}^{i} & =u_{j}^{i} \omega^{j}, \\
\bar{\pi}^{i} & =r_{j}^{i} \pi^{j}+s_{j}^{i} \omega^{j} .
\end{aligned}
$$

Taking the bilinear covariants of the above equations and comparing the coefficients on both sides, we obtain

$$
\begin{aligned}
r_{j}^{i} & =\frac{1}{u} s_{i}^{i}, \\
\frac{d u_{j}^{i}}{d x} & =u s_{j}^{i}+\frac{1}{2} u u_{j}^{k}\left(\frac{\partial \bar{F}^{i}}{\partial \bar{p}^{k}}+\delta_{k}^{i} \bar{H}\right)-\frac{1}{2} u_{l}^{i}\left(\frac{\partial F^{l}}{\partial p^{j}}+\delta_{j}^{l} H\right),
\end{aligned}
$$

(9) $\frac{d}{d x}\left(\frac{1}{u} u_{i}^{i}\right)=\frac{1}{2} u_{i}^{l}\left(\frac{\partial \bar{F}^{i}}{\partial \bar{p}^{l}}-\delta_{l}^{i} \bar{H}\right)-\frac{1}{2 u} u_{l}^{i}\left(\frac{\partial F^{l}}{\partial p^{i}}-\delta_{j}^{l} H\right)+s_{i}^{i}$,

$$
\begin{aligned}
\frac{d}{d x} s_{k}^{i}= & u u_{k}^{i} \bar{Q}_{j}^{i}-\frac{1}{u} u_{j}^{i} Q_{k}^{i}+\frac{u}{2} s_{k}^{j}\left(\frac{\partial \bar{F}^{i}}{\partial \bar{p}^{j}}-\delta_{j}^{i} \bar{H}\right) \\
& -\frac{1}{2} s_{j}^{i}\left(\frac{\partial F^{i}}{\partial p^{k}}+\delta_{k}^{i} H\right) .
\end{aligned}
$$

Let $v_{j}^{q}$ and $s$ be defined by the equations

$$
u_{j}^{i} v_{k}^{j}=\delta_{k}^{i}, \quad s=s_{j}^{i} v_{i}^{j},
$$

$\delta_{k}^{b}$ being the well known Kronecker symbol. Then the first three sets of equations of (9) give

$$
s_{i}^{i}=\frac{s}{n} u_{i}^{j}
$$

while the last set gives, after the elimination of $d s_{4}^{3} / d x$,

$$
u_{i}^{i}\left(\frac{d s}{d x}+\frac{s^{2} u}{n}+s u \bar{H}\right)=u u_{j}^{k} \bar{Q}_{k}^{i}-\frac{1}{u} u_{k}^{i} Q_{j}^{k}
$$

Multiplying (11) by $v_{i}^{j}$ and contracting, we get

$$
d s / d x+s^{2} u / n+s u \bar{H}=0,
$$

since $\bar{Q}_{i}^{\prime}=Q_{i}^{i}=0$. Finally, we have $\bar{Q}_{j}^{\sharp}=\left(1 / u^{2}\right) u_{k}^{i} Q_{l}^{k} v_{j}^{l}$, which shows 
that the quantities $Q_{j}^{f}$ undergo a linear transformation. The vanishing of $Q_{j}^{q}$ has therefore a geometrical meaning.

The quantities $Q_{j}^{q}$ differ slightly from similar ones employed by Douglas. ${ }^{5}$ The condition $Q_{t}^{i}=0$ makes them obey a simpler transformation law.

3. Conditions of intersection of paths. Let the system of finite equations defining the family of paths be

$$
y^{i}=G^{i}\left(x, C^{\alpha}\right), \quad \alpha=1, \cdots, 2 n .
$$

We can consider $C^{\alpha}$ as coordinates in a $2 n$-dimensional space $R_{2 n}$ whose points are our paths. Paths are said to be neighboring if the corresponding points are infinitely near. Let $\Gamma$ and $\Gamma^{\prime}$ be two neighboring paths defined by the parameters $C^{\alpha}$ and $C^{\alpha}+\delta C^{\alpha}$ respectively. In order that they intersect, we must have

$$
\delta y^{i}=\left(\partial G^{i} / \partial C^{\alpha}\right) \delta C^{\alpha}=0, \quad \alpha=1, \cdots, 2 n .
$$

The system of $n-1$ equations obtained by eliminating $x$ from (13) gives the conditions of intersection. The $n-1$ equations are independent, otherwise the paths intersecting a given path would depend on more than $n+1$ essential parameters, which case is excluded.

We proceed to show how the conditions of intersection can be treated most simply by our symbolism. Let us introduce two differential operations $d$ and $\delta$, the operation $d$ denoting the variation along the path, while $\delta$ denotes the change from one path to the other. By definition they satisfy the conditions

$$
d x \neq 0, \quad d C^{\alpha}=0, \quad \delta x=0, \quad \delta C^{\alpha} \neq 0,
$$

so that

$$
\begin{aligned}
& \omega^{i}(d)=0, \quad \pi^{i}(d)=0, \\
& \omega^{i}(\delta)=\delta y^{i}=\left(\partial G^{i} / \partial C^{\alpha}\right) \delta C^{\alpha}, \\
& \pi^{i}(\delta)=\delta p^{i}-\frac{1}{2}\left(\frac{\partial F^{i}}{\partial p^{j}}+\delta_{j}^{i} H\right) \delta y^{j} \\
&=\left\{\frac{\partial}{\partial C^{\alpha}}\left(\frac{\partial G^{i}}{\partial x}\right)-\frac{1}{2}\left(\frac{\partial F^{i}}{\partial p^{j}}+\delta_{j}^{i} H\right) \frac{\partial G^{j}}{\partial C^{\alpha}}\right\} \delta C^{\alpha}, \\
& \alpha=1, \cdots, 2 n .
\end{aligned}
$$

According to the theory of completely integrable Pfaffian systems, the last $2 n$ equations can be solved in terms of $\delta C^{\alpha}$, thus expressing $\delta C^{\alpha}$ as linear combinations of $\omega^{i}(\delta)$ and $\pi^{i}(\delta)$. 
Suppose that the system giving the conditions of intersection consists only of Monge's equations of the second degree:

$$
A_{\alpha \beta}^{\alpha}\left(C^{\gamma}\right) \delta C^{\alpha} \delta C^{\beta}=0, \quad \alpha, \beta, \gamma=1, \cdots, 2 n ; \epsilon=1, \cdots, n-1 .
$$

We express the left-hand side in terms of $\omega^{i}(\delta)$ and $\pi^{i}(\delta)$, getting

$$
\begin{array}{r}
A_{\alpha \beta}^{\dot{e}}\left(C^{\gamma}\right) \delta C^{\alpha} \delta C^{\beta}=a_{i j}^{i} \omega^{i}(\delta) \omega^{j}(\delta)+b_{i j}^{e} \omega^{i}(\delta) \pi^{j}(\delta)+e_{i j}^{i} \pi^{j}(\delta) \pi^{j}(\delta), \\
\alpha, \beta, \gamma=1, \cdots, 2 n, \epsilon=1, \cdots, n-1,
\end{array}
$$

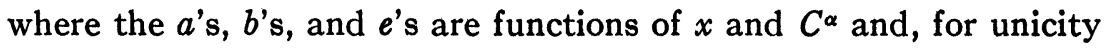
of the coefficients, we can assume

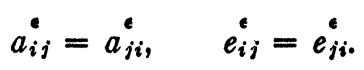

Since (15) is evidently satisfied when $\omega^{i}(\delta)=0, e_{i j}^{i}$ must vanish, so that (16) becomes

$$
A_{\alpha \beta}^{\dot{e}}\left(C^{\gamma}\right) \delta C^{\alpha} \delta C^{\beta}=a_{i j}^{e} \omega^{i}(\delta) \omega^{j}(\delta)+b_{i j}^{e} \omega^{i}(\delta) \pi^{j}(\delta) .
$$

The left-hand side of the preceding equation is independent of $x$, so that the same is true of the right-hand side. Thus we have

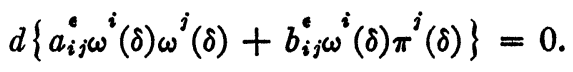

To expand (18) we notice that from (5) we have

$$
\begin{aligned}
d \omega^{i}(\delta) & =d x \pi^{i}(\delta)+\left(\partial F^{i} / \partial p^{k}+\delta_{k}^{i} H\right) d x \omega^{k}(\delta) / 2, \\
d \pi^{i}(\delta) & =Q_{j}^{i} d x \omega^{j}(\delta)+\left(\partial F^{i} / \partial p^{j}-\delta_{j}^{i} H\right) d x \pi^{j}(\delta) / 2 .
\end{aligned}
$$

Substituting these expressions into (18) we get

$$
\begin{aligned}
&\left\{d a_{i k}^{e}+\right. {\left.\left[a_{i j}^{e}\left(\frac{\partial F^{j}}{\partial p^{k}}+\delta_{k}^{i} H\right)+b_{i j}^{e} Q_{k}^{i}\right] d x\right\} \omega^{i}(\delta) \omega^{k}(\delta)+b_{i j}^{e} d x \pi^{i}(\delta) \pi^{j}(\delta) } \\
&+\left\{d b_{i k}^{e}+\frac{1}{2}\left(4 a_{i k}^{e}+b_{i j}^{e} \frac{\partial F^{j}}{\partial p^{k}}+b_{j k}^{e} \frac{\partial F^{j}}{\partial p^{i}}\right) d x\right\} \omega^{i}(\delta) \pi^{k}(\delta)=0, \\
& \epsilon=1,2, \cdots, n-1 .
\end{aligned}
$$

It follows that

$$
\begin{gathered}
b_{i j}^{e}+b_{j i}^{\dot{c}}=0, \\
d b_{i k}^{e}+\frac{1}{2}\left(4 a_{i k}^{e}+b_{i j}^{e} \frac{\partial F^{j}}{\partial p^{k}}+b_{j k}^{e} \frac{\partial F^{j}}{\partial p^{i}}\right) d x=0
\end{gathered}
$$

$$
\begin{aligned}
2 d a_{i k}^{\cdot}+\left\{a _ { i j } ^ { i } \left(\frac{\partial F^{j}}{\partial p^{k}}\right.\right. & \left.+\delta_{k}^{i} H\right) \\
& \left.+a_{k j}^{i}\left(\frac{\partial F^{j}}{\partial p^{i}}+\delta_{i}^{j} H\right)+b_{i j}^{i} Q_{k}^{i}+b_{k j}^{i} Q_{i}^{i}\right\} d x=0 .
\end{aligned}
$$


On account of the symmetry of $a_{\mathfrak{i}}^{\mathfrak{e}}$ and the skew-symmetry of $b_{\mathfrak{k} k}^{\mathfrak{e}}$ in the lower indices the second set of equations of (20) gives

$$
a_{i k}^{e}=0 \text {. }
$$

The last two equations of (20) then become

$$
\begin{gathered}
d b_{i k}^{e}+\frac{1}{2}\left(b_{i j}^{e} \frac{\partial F^{j}}{\partial p^{k}}+b_{j k}^{e} \frac{\partial F^{j}}{\partial p^{i}}\right) d x=0, \\
b_{i j}^{e} Q_{k}^{j}+b_{k j}^{e} Q_{i}^{j}=0 .
\end{gathered}
$$

As a conclusion of our discussions we see that the existence of $b_{i j}^{e}$ satisfying (22) is a necessary condition for the family of paths to have the prescribed property, that is, the conditions of intersection be given by a system of Monge's equations of the second degree.

4. The main theorem. Our main theorem can be stated as follows:

THEOREM. The conditions of intersection of neighboring paths of the family defined by (2) consist only of Monge's equations of the second degree if and only if all the components of the tensor $Q_{j}^{i}$ vanish.

In order to prove the theorem we first establish two simple lemmas.

LEMMA 1. Let $K^{\circ}$ be $n-1$ skew-symmetric matrices of order $n$ whose first rows are linearly independent, and $R$ be any matrix of the same order. Then the equations

$$
K^{\bullet} R-R^{\prime} K^{\epsilon}=0, \quad \epsilon=1, \cdots, n-1, R^{\prime}=\text { transpose of } R,
$$

imply that $R$ is a scalar matrix.

Proof. By hypothesis, there exist $n-1$ linear combinations $C^{\epsilon}$ of $K^{\bullet}$ having their first rows identical to

$$
(0,1,0, \cdots, 0,0),(0,0,1, \cdots, 0,0), \cdots,(0,0, \cdots, 0,1)
$$

respectively. As the $K^{\prime}$ 's satisfy (23), so do all the $C$ 's, that is,

$$
C^{\epsilon} R-R^{\prime} C^{\epsilon}=0 .
$$

By actually writing out the elements of the matrices, we can justify this lemma by a short calculation.

Lemma 2. Let $b_{i j}^{i} x^{i} y^{i}=0, \epsilon=1, \cdots, n-1$, be $n-1$ independent equations in $x^{i}, y^{j}$, with skew-symmetric coefficients

$$
b_{i j}^{e}+b_{j i}^{e}=0 .
$$

Let $Q$ be a square matrix satisfying 


$$
B^{\bullet} Q-Q^{\prime} B^{\bullet}=0
$$

where

$$
B^{\epsilon}=\left(b_{i j}^{e}\right)
$$

Then $Q$ is a scalar matrix.

Proof. We can find $x_{0}^{i}$ such that the $n-1$ linear equations in $y^{i}$,

$$
b_{i j}^{i} x_{0}^{i} y^{j}=0
$$

are linearly independent. Let $P$ be any nonsingular matrix with $x_{0}^{4}$ as its first row. The first rows of $P B^{*}$ are

$$
\left(b_{i 1}^{e} x_{0}^{i}, b_{i 2}^{e} x_{0}^{i}, \cdots, b_{i n}^{e} x_{0}^{i}\right),
$$

respectively, and hence are linearly independent. It is easy to see that the matrices $P B^{\prime} P^{\prime}$ satisfy all the hypotheses for $K^{\bullet}$ in Lemma 1 . By writing (24) in the form

$$
\left(P B^{\bullet} P^{\prime}\right)\left(P^{\prime-1} Q P^{\prime}\right)-\left(P^{\prime-1} Q P^{\prime}\right)^{\prime}\left(P B^{\bullet} P^{\prime}\right)=0
$$

it follows that $P^{\prime-1} Q P^{\prime}$, and hence $Q$, is a scalar matrix.

Proof of The main Theorem. Suppose that the conditions of intersection of neighboring paths of the family consist only of Monge's equations of the second degree. There exist $b_{i j}^{\epsilon}(\epsilon=1, \cdots, n-1)$ satisfying (22) and having the property that the $n-1$ equations

$$
b_{i j}^{\epsilon} \omega^{i}(\delta) \pi^{j}(\delta)=0
$$

are independent. From Lemma 2 it follows that

$$
Q_{i}^{i}=(1 / n) \delta_{j}^{i} Q_{l}^{l}=0 \text {. }
$$

Conversely, suppose $Q_{j}^{i}=0$. We regard $b_{i k}^{e}=-b_{k i}^{e}$ as functions of $x$, $C^{\alpha}$ and consider the system of $(n-1) n / 2$ partial differential equations

$$
\partial b_{i k} / \partial x+(1 / 2)\left(b_{i j} \partial F^{j} / \partial p^{k}+b_{j k} \partial F^{j} / \partial p^{i}\right)=0,
$$

where

$$
\frac{\partial F^{j}}{\partial p^{l}}=\frac{\partial F^{j}\left(x, G^{m}, \partial G^{m} / \partial x\right)}{\partial p^{l}} .
$$

Let $b_{i j}^{e}$ be $n-1$ sets of integrals satisfying the initial conditions that all $\left(b_{i j}\right)_{x=x_{0}}$ vanish except

$$
\left(b_{1 \epsilon+1}^{e}\right)_{x=x_{0}}=-\left(b_{\epsilon+1}^{e} 1\right)_{x=x_{0}}=1 .
$$


The quantities $b_{i j}^{e}$ so obtained not only satisfy (22) but also possess the property that the equations

$$
b_{i j}^{\cdot i} \omega^{i}(\delta) \pi^{j}(\delta)=0
$$

are independent. That these equations give the conditions of intersection of neighboring paths follows from our discussion. Therefore the theorem is proved.

National Southwest Associated University, Kunming, China 